ZOOLOGIA 32 (2): 123-138, April 2015

http://dx.doi.org/10.1590/S1984-46702015000200003

\title{
Two new tiny Nemesiidae species from Reserva Biológica do Tinguá, Rio de Janeiro, Brazil (Araneae: Mygalomorphae)
}

\author{
Rafael P. Indicatti ${ }^{1,4}$, Elaine Folly-Ramos², André B. Vargas ${ }^{3}$, \\ Sylvia M. Lucas ${ }^{1} \&$ Antonio D. Brescovit ${ }^{1}$
}

\begin{abstract}
'Laboratório Especial de Coleções Zoológicas, Instituto Butantan. Avenida Vital Brazil 1500, 05503-900 São Paulo, SP, Brazil. 'Laboratório de Ecologia Animal, Departamento de Engenharia e Meio Ambiente, Centro de Ciências Aplicadas e Educação, Universidade Federal da Paraíba. Rua Manuel Gonçalves S/N, 58297-000 Rio Tinto, PB, Brazil. ${ }^{3}$ Centro Universitário de Volta Redonda. Avenida Paulo Erlei Alves Abrantes 1325, 27240-560 Volta Redonda, RJ, Brazil. ${ }^{4}$ Corresponding author. E-mail: indicatti@gmail.com
\end{abstract}

\begin{abstract}
Two new Nemesiidae species from Reserva Biológica do Tinguá, Rio de Janeiro, Brazil are described. Acanthogonatus minimus sp. nov. differs from the remaining species of the genus by the male palpal bulb, which has very long and twisted embolus, ca. 2/3 the length of the palpal tibia, long and twisted spermathecae, anterior eye row recurved and fovea T-shaped. Chaco tingua sp. nov. differs from the remaining species of the genus by the retrolateral megaspine on tibia I, palpal embolus tip hook-shaped, inferior tarsal claw on all legs, absence of pubescence on the carapace and legs. Both species were collected with Winkler extractors in leaf litter. They are the smallest specimens in their respective genera and also among the world Nemesiidae described to date. The smallest male of $A$. minimus sp. nov. measures $4.22 \mathrm{~mm}$ and of $C$. tingua sp. nov. measures $3.85 \mathrm{~mm}$. Data on the phenology of $C$. tingua sp. nov. is presented.
\end{abstract}

KEY WORDS. Anaminae; Diplothelopsinae; Mata Atlântica; phenology; spider.

The Nemesiidae are the second most speciose family of Mygalomorphae, comprising 374 species in 44 genera (Platnick 2014). The Nemesiidae include medium sized spiders, for example Prorachias bristowei Mello-Leitão, 1924 (length $38.7 \mathrm{~mm}$ ) and Lycinus caldera Goloboff, 1995 (35.9 mm), and small ones, e.g., Hermacha anomala (Bertkau, 1880) $(8.6 \mathrm{~mm})$ and Flamencopsis minima Goloboff, 1995 (10.7 mm) (BERTKAU 1880, Goloboff 1995, LuCAs et al. 2005). The Nemesiidae are distributed world-wide and are divided into six subfamilies (RAven 1985). Of these, four occur in the Neotropical region: Anaminae, with three genera: Acanthogonatus Karsch, 1880, Longistylus Indicatti \& Lucas, 2005 and Hermacha Simon, 1889; Diplothelopsinae, with five genera: Chaco Tullgren, 1905, Chilelopsis Goloboff, 1995, Diplothelopsis Tullgren, 1905, Flamencopsis Goloboff, 1995 and Lycinus Thorell, 1894; Nemesiinae with one genera: Mexentypesa Raven, 1987; and Pycnothelinae with nine genera: Bayana Pérez-Miles et al., 2014, Hermachura Mello-Leitão, 1923, Neostothis Vellard, 1925, Prorachias Mello-Leitão, 1924, Psalistopoides Mello-Leitão, 1934, Pselligmus Simon, 1892, Pycnothele Chamberlin, 1917, Rachias Simon, 1892 and Stenoterommata Holmberg, 1881 (RAVEN 1985, 1987, Goloboff 1995, Indicatti \& Lucas 2005, Lucas \& IndicatTi 2006, Lucas et al. 2008, Passanha et al. 2014, Pérez-Miles et al. 2014). In this study we deal with species that belong to Acanthogonatus and Chaco.
Acanthogonatus was established by KaRsCH (1880), with the type species, $A$. francki Karsch, 1880, based on a female from Chile. The genus currently includes 28 species, distributed mostly in the Occidental portion of South America, mainly in Chile and Argentina, and includes 25 species (Goloboff 1995). In the Oriental portion, only A. ericae Indicatti et al., 2008 and A. tacuariensis (Pérez-Miles \& Capocasale, 1982) are recorded, both in southern Brazil and the second also in Uruguay (PérezMiles \& Capocasale 1982, Goloboff 1995, Indicatti et al. 2008).

Chaco was established by Tullgren (1905), with the type species, C. obscura Tullgren, 1905, based on a male from Salta, Argentina. The genus currently includes 11 species, six from Argentina, two from Chile, two from Uruguay and one from Brazil (Goloboff 1995, Montes de Oca \& Pérez-Miles 2013, Ferretti 2014).

In an inventory using Winkler extractors in leaf litter in the Reserva Biológica do Tinguá, Rio de Janeiro, Brazil, representatives of two new Nemesiidae species were collected and are herein described: Acanthogonatus minimus sp. nov. and Chaco tingua sp. nov. In addition, data on the phenology of $C$. tingua sp. nov. is provided.

\section{MATERIAL AND METHODS}

The material examined is deposited in the following institutions (abbreviation and curator in parentheses): Instituto

2015 | Sociedade Brasileira de Zoologia | www.sbzoologia.org.br | www.scielo.br/zool All content of the journal, except where identified, is licensed under a Creative Commons attribution-type BY-NC. 
Butantan, São Paulo (IBSP, A.D. Brescovit), Museu Nacional do Rio de Janeiro, Rio de Janeiro (MNRJ, A.B. Kury), Museu de Zoologia da Universidade de São Paulo, São Paulo (MZSP, R. Pinto da Rocha), and Museu de Ciências Naturais, Fundação Zoobotânica do Rio Grande do Sul, Porto Alegre (MCN, R. Ott). The format of the descriptions follows LuCAS \& INDICATTI (2010). The terminology for general structures follows Goloboff (1995). Spine notation follows Petrunkevitch (1925). All measurements are in millimeters $(\mathrm{mm})$ and were taken with a stereomicroscope equipped with a millimetric ocular lens. The lengths of leg segments were measured between joints, in dorsal view. Total body length includes the chelicerae but not the pedicel and spinnerets. Spermathecae were dissected and immersed in enzyme (Ultrazyme ${ }^{\circledR}$ ) during 72 hours for soft tissue digestion to allow observation of internal structures. The material for scanning electronic microscopy (SEM) was cleaned in an ultrasonic cleaner USC 700, Thornton Unique, for six minutes, and was gradually dehydrated through immersion in increasingly concentrated (from 80 to 100\%) ethanol for approximately eight hours. After dehydration it was critical-point dried. All the material used in SEM was fixed to stubs with doublefaced adhesive copper tape and sputter-coated with gold. Images were taken under high vacuum with a FEI Quanta 250 SEM at the Instituto Butantan. Pictures were taken with a Leica DFC500 digital camera mounted on a Leica MZ16A stereomicroscope, the extended focal range images were composed with Leica Application Suite version 2.5.0. Abbreviations: (AME) anterior median eyes, (ALE) anterior lateral eyes, (PLE) posterior lateral eyes, (PME) posterior median eyes, (PMS) posterior median spinnerets, (PLS) posterior lateral spinnerets, (STC) superior tarsal claws, (ITC) inferior tarsal claw; spines: (d) dorsal, (v) ventral, (p) prolateral, (r) retrolateral, (ap) apical, (VP) ventro-prolateral, (VR) ventro-retrolateral.

Ecological data were obtained at Reserva Biológica do Tinguá, located at Serra do Mar, municipality of Nova Iguaçu, state of Rio de Janeiro (southeastern Brazil). The Reserva has an area of 26,260 ha, and altitudinal gradient from 50-1600 $\mathrm{m}$ a.s.l. The climate there is classified as ' $\mathrm{C} w b$ ' in the Köppen system, temperate humid highland tropical (IBAMA 2006). This type of climate has a short, poorly defined dry season in July and August, averaging $20^{\circ} \mathrm{C}$, and a rainy season in December, January and February averaging $27^{\circ} \mathrm{C}$. The annual precipitation is $2,099.3 \mathrm{~mm}$, with the wettest months in December and January (IBAMA 2006). The vegetation is classified as Dense Ombrophylous Forest (Atlantic Rainforest), with large structural variation due to its declivity (IBAMA 2006). The study location, known as Barrelão ( $\left.22^{\circ} 34^{\prime} 28.9^{\prime} \mathrm{S}, 43^{\circ} 24^{\prime} 57.6^{\prime} \mathrm{W}\right)$, close to $400 \mathrm{~m}$ a.s.l. altitude is classified as Dense Ombrophylous Submontane Forest (Orsolon-Souza et al. 2011: fig. 1 A-C).

Samples were collected in the middle of each of the four seasons of 2002: summer (February 02-04); autumn (May 1517); winter (June 05-07); spring (October 20-22). During each field excursion 25 points were marked along a 1,200 m transect, and at each point we stretched two perpendicular, $25 \mathrm{~m}$ lines; one to the left and the other one to the right. At the end of each line we delimited a $1 \mathrm{~m}^{2}$ plot, totaling 50 plots. Each sample refers to one square meter of leaf litter, which was removed and sieved in a $5 \mathrm{~mm}$ mesh and soon after placed into the Winkler extractor where it remained for 48 hours. This procedure was adapted from Delabie et al. (2000) and OrsolonSouzA et al. (2011). Since abundance data did not meet the assumptions of parametric tests, the Kruskal-Wallis (KW) nonparametric analysis of variance was used for detecting betweenseasons differences in abundance of males, females and juveniles separately (ZAR 1999).

\section{TAXONOMY}

\section{Nemesiidae Simon, 1889 Anaminae Simon, 1889 Acanthogonatus Karsch, 1880}

Identification key for Acanthogonatus updated from Goloboff (1995)

\section{Males}

Male of A. alegre Goloboff, 1995, A. brunneus (Nicolet, 1849), A. incursus (Chamberlin, 1916), A. juncal Goloboff, 1995, A. mulchen Goloboff, 1995, A. parana Goloboff, 1995, A. peniasco Goloboff, 1995, A. tolhuaca Goloboff, 1995 and A. vilches Goloboff, 1995 are unknown.

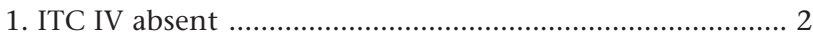

1'. ITC IV present ........................................................... 14

2. Apophysis with two apical laminar spines on the same base (Goloboff 1995: figs. 75-78); palpal tibia with two dorsal sinuous long setae (Goloboff 1995: figs. 9, 79F, 80E, 89C); bulb with no keels, or with lateral keels .......................... 3

2 '. No tibial apophysis of any kind; ITC I absent; forests in southern Chile and Argentina......A. confusus Goloboff, 1995

3. Bulb with a lateral keel forming a concavity (GoLoboff 1995: fig. 81B, C); central Chile (regions IV, V).....

A. huaquen Goloboff, 1995

3'. Bulb different

.. 4

4. Dorsal abdomen yellowish with a chevron (Goloвoff 1995: figs. 98A, 100A); apical article of PLS short, triangular; Argentina (dry regions of Patagonia) and southern Chile.. .

4 '. Dorsal abdomen with pattern formed by numerous mottles (GoLoboff 1995: figs. 84A, 103A, 109B); apical article of PLS longer, digitiform; Argentina (north of Patagonia) ........ 8

5. Large spiders (about $20 \mathrm{~mm}$ total length, carapace over $9 \mathrm{~mm}$ ); bulb with two lateral flanges delimiting concave triangular area (Goloboff 1995: fig. 98C); palpal tibia rather elongate (Goloboff 1995: fig. 98B) ....... A. patagonicus (Simon, 1905) 
5'. Medium to small spiders (total length $15 \mathrm{~mm}$ or smaller, carapace below $6 \mathrm{~mm}$ ); bulb variable; palpal tibia shorter...

... 6

6. Bulb with low lateral keels (or with single keel) (Goloboff 1995: figs. 101A, B, 102A, B); metatarsi I with at least 1 or 2 ventral spines

6'. Bulb with more developed lateral keels (GoloвоғF 1995: fig. 100C, D); metatarsi I with no ventral spines A. notatus (Mello-Leitão, 1940)

7. Bulb with a single keel (GoLoboff 1995: fig. 102A, B); patella III with 1-1-1; small size (carapace length about $3.5 \mathrm{~mm}$ )...... A. birabeni Goloboff, 1995

7'. Bulb with a double keel (GoLoboff 1995: figs. 101A, B); patella III with only 0-0-1; slightly larger (carapace length about $5 \mathrm{~mm})$ A. chilechico Goloboff, 1995

8. Dorsal abdomen blackish with yellowishwhitish oblique lines; bulb with widened, flanged tip (Goloboff 1995: fig. 87B); central-southern Chile (regions IV-VIII)

A. pissii (Simon, 1889)

8 '. Dorsal abdomen with only white dots not forming continuous lines, or mottled; bulb with narrower tip, flanged in the base or without flanges ... 9

9. Bulb with no keels (Goloboff 1995: fig. 89E, F); total length ca. 13 mm; central Argentina (Córdoba, San Luis, San Juan, and Buenos Aires) A. centralis Goloboff, 1995

9'. Bulb with serrated, curved and long embolus (GoLовоғF 1995: figs. 83B, 85C; INDiCATTI et al. 2008: fig. 17); central Chile, Uruguay and southern Brazil ... 10

10. Metatarsus I slightly curved downward; central Chile ..... A. quilocura Goloboff, 1995

10. Metatarsus I straight 11

11. Presence of a flange on palpal embolus apex (InDiCatTi et al. 2008: figs. 17, 21); southern Brazil A. ericae

11'. Absence of a flange on palpal embolus apex (Goloboff 1995: fig. 85C); Uruguay and southern Brazil ...... A. tacuariensis

12. Bulb with three perpendicular flanges along embolus (Goloboff 1995: fig. 95D, E) A. francki

$12^{\prime}$. Bulb different 13

13. Bulb with a winglike projection (GoLobofF 1995: fig. 96D); PLS apical segment digitiform ... A. recinto Goloboff, 1995

13'. Bulb smooth (GoloвоғF 1995: fig. 99D, E); PLS apical segment triangular; Argentina (Patagonia)

A. fuegianus (Simon, 1902)

14. Anterior tibia with dense prolateral shield of setae (GolobofF 1995: figs. 103C) 15

$14^{\prime}$. Anterior tibia without such shield 17

15. Palpal tibia widest in basal third, and then uniformly tapering (GoLobofF 1995: fig. 103D); retrolateral thicker setae present along apical 2/3 of article 16

$15^{\prime}$. Palpal tibia of uniform width along medial half, more abruptly narrowed in the apical third (Goloboff 1995: fig. 107A); retrolateral thicker setae present only on apical third ......... A. hualpen Goloboff, 1995

16. Bulb with its basal portion rounded, abruptly tapered to form embolus base (GoLOBOFF 1995: fig. 108A); basal portion of the bulb duct strongly sinuous (GоLовоғғ 1995: fig. 108B) A. patagallina Goloboff, 1995

16 '. Bulb with its basal portion not rounded, tapering more gradually to form embolus base (Goloboff 1995: fig. 103F); basal portion of the bulb duct little sinuous (Goloboff 1995: fig. 103E) .......................... A. nahuelbuta Goloboff, 1995

17. Tibia I with a strong apical retrolateral megaspine (Fig. 5); palpal bulb with twisted embolus (Figs. 2, 4); total length less than $5 \mathrm{~mm}$; Brazil (Rio de Janeiro) ........ A. minimus sp. nov.

$17^{\prime}$. Tibia I with no retrolateral megaspine; palpal bulb with slightly curved embolus; total length more than $13 \mathrm{~mm}$............. 18

18. No apophysis on anterior tibia A. confusus

18'. Low prolateral tibial apophysis, bearing two spines on common base 19

19. Palpi long, about three times of the cymbium length (GoLOBOfF 1995: fig. 79F); embolus ca. half length of palpal bulb (GoLOBOFF 1995: fig. 79G, H) .............. A. subcalpeianus (Nicolet, 1849)

$19^{\prime}$. Palpi normal, about two times of the cymbium length; embolus ca. 2/3 length of palpal bulb (Goloboff 1995: figs. 92A, B, 93A, B) ............ A. campanae (Legendre \& Calderón, 1984)

\section{Females}

Female of A. birabeni, A. chilechico and A. patagallina are unknown.

1. Claw tufts present; Peru A. incursus

$1^{\prime}$. Claw tufts absent ............................................................. 2

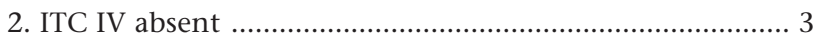

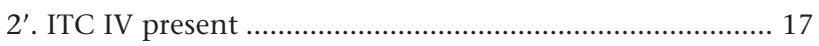

3. Patella IV with 1-1-1 ........................................................ 4

3'. Patella IV with 0-0-1 or (more often) no spines at all ..... 7

4. Spermathecae thick, sclerotized (GolobofF 1995: fig. 97)........... A. peniasco

4'. Spermathecae slightly sclerotized ……........................... 5

5. Spermathecae cactus like (GoloвоғF 1995: fig. 89A); Argentina A. centralis

5'. Spermathecae different; Chile .. 6

6. Spermathecae with short duct, slightly curved from the internal side (Goloboff 1995: fig. 95A) A. francki

6 '. Spermathecae without basal dome, long twisted receptaculum duct (Goloboff 1995: fig. 96A) A. recinto 
7. Apical article of PLS triangular. 8

7'. Apical article of PLS longer, digitiform 10

8. Spermathecae branched (bi- or trifurcated) (GoloвоғF 1995: fig. 99A) A. fuegianus

8'. Spermathecae with a basal mound or protuberance ........ 9

9. Small spiders (total length about 12 , carapace length 5-6 $\mathrm{mm}$ ); carapace slightly patterned, with margins and medial line lighter; lateral stripes of dorsal abdominal chevron conspicuous (GoLOBOFF 1995: fig. 100A); general coloration yellowish A. notatus

9'. Larger spiders (total length well over $20 \mathrm{~mm}$, carapace length $10 \mathrm{~mm}$ or more); carapace not appreciably patterned; dorsal abdominal pattern limited mostly to cardiac area (Goloboff 1995: fig. 98A); general coloration brown with golden hairs A. patagonicus

10. Spermathecae with a main branch and a lateral secondary internal branch arising from middle of main branch (Goloboff 1995: figs. 89A, 9 1B) 11

10. Spermathecal duct arising from the inner side of basal mound or protuberance (GoLoBofF 1995: figs. 83A, 84B, 86A

11. Main spermathecal branch widened distally (GoLoBofF 1995: fig. 89A); central Argentina A. centralis

11 '. Main spermathecal branch not widened distally (Goloboff 1995: fig. 91B); eastern Argentina (Entre Rios) .. A. parana

12. Dorsal abdomen blackish with yellow-white oblique lines; spermathecae with low basal dome (GolobofF 1995: fig. 86A); central and southern Chile (regions IV-VIII) .......... A. pissii

12 '. Dorsal abdomen with white dots or spots not forming continuous lines, or yellowish with darker mottles ..... 13

13. Small spiders (total length about $10 \mathrm{~mm}$, carapace $4 \mathrm{~mm}$ or smaller); spermathecae weakly sclerotized, very short duct (Goloboff 1995: fig. 84B); patella III with p0-1-1 spines ... A. juncal

13'. Larger (total length $20 \mathrm{~mm}$ or more, carapace $8 \mathrm{~mm}$ or more); patella III with p1-1-1 spines.... 14

14. Spermathecae with blunt basal mound, and duct strongly curved at the base (Golobofr 1995: fig. 83A) .. A. quilocura

$14^{\prime}$. Spermathecae with basal mound tapering more gradually 15

15. Carapace brownish, dorsal abdomen irregularly mottled; with (weak) rastellum; spermathecae with basal dome narrow, duct strong curvate to inner side (Goloboff 1995: fig. 81A); central Chile A. huaquen

15'. Carapace reddish, dorsal abdomen with oblique lines of dots; rastellum absent. 16

16. Spermathecae with short copulatory ducts, arising from basal dome side (GolobofF 1995: fig. 85A); Uruguay and southern Brazil.... A. tacuariensis
16 '. Spermathecae with short copulatory ducts, arising from the basal dome apex (INDicatTI et al. 2008: fig. 19); southern Brazil ..... A. ericae

17. ITC I present …........................................................ 18

$17^{\prime}$. ITC I absent …......................................................... 25

18. Spermathecae very long, twisted (Fig. 6); fovea T-shaped (Fig. 11); Brazil (Rio de Janeiro) .......... A. minimus sp. nov.

18 '. Spermathecae short, not twisted; fovea normal, procurved

19

19. Spermathecae two wide (but flat) plates ....................... 20

19'. Spermathecae different ..................................................... 21

20. Sternum long (Goloboff 1995: fig. 112C); spermathecae fused, without copulatory duct (Goloboff 1995: fig. 112B), with shallow medial notch A. mulchen

20'. Sternum normal (Goloboff 1995: fig. 112C) ................ 22

21. color uniform blackish; patella III with p0-1-1 spines ...... A. tolhuaca

$21^{\prime}$. Color brownish, with mottled abdomen; patella III with p0-1-1 or p1-1-1 spines A. brunneus

22. Spermathecae strongly sclerotized, with a wide cavity, opening through wide passage .................................... 23

22 '. Spermathecae weakly sclerotized, flat and with no appreciable cavity; duct opening not conspicuous ................. 24

23. Spermathecae finger like, strongly curved (Goloboff 1995: fig. 106A-C) ........................ A. nahuelbuta and A. hualpen

23'. Spermathecae wider, shaped like two pockets (Golobofr 1995: fig. 109D)

A. vilches

24. Carapace patterned, legs ringed; spermathecae with short copulatory ducts, arising from the external side of basal dome (Goloboff 1995: fig. 79E) ................ A. subcalpeianus

24 '. Carapace and legs uniform brown; spermathecae single, undivided (Goloboff 1995: fig. 92C); central Chile (regions IV, V) ........................................................ A. campanae

25. Epigastrium posteriorly produced (GolobofF 1995, fig. 94B); spermathecae long (Goloboff 1995: 94A); northern Chile (Region II)

A. alegre

25'. Epigastrium normal; spermathecae shorter (GoloвоғF 1995: fig. 80A-C); southern Chile (Regions VIII-X) and Argentina (Neuquén) A. confusus

\section{Acanthogonatus minimus sp. nov.} Figs. 1-30

Types. Male holotype from BraziL, Rio de Janeiro: Nova Iguaçu (Reserva Biológica do Tinguá, $22^{\circ} 34^{\prime} 28.9^{\prime}$ S, $43^{\circ} 24^{\prime} 57.6^{\prime} \mathrm{W}$, $400 \mathrm{~m}$ a.s.l.), 2002, E. Folly Ramos et al. coll. (IBSP 166089). Paratypes with the same data as holotype, female (IBSP 166090); one male (IBSP 166091); female (IBSP 166092); female (MNRJ 6803); female (MZSP 67296); female (MCN 52059). 

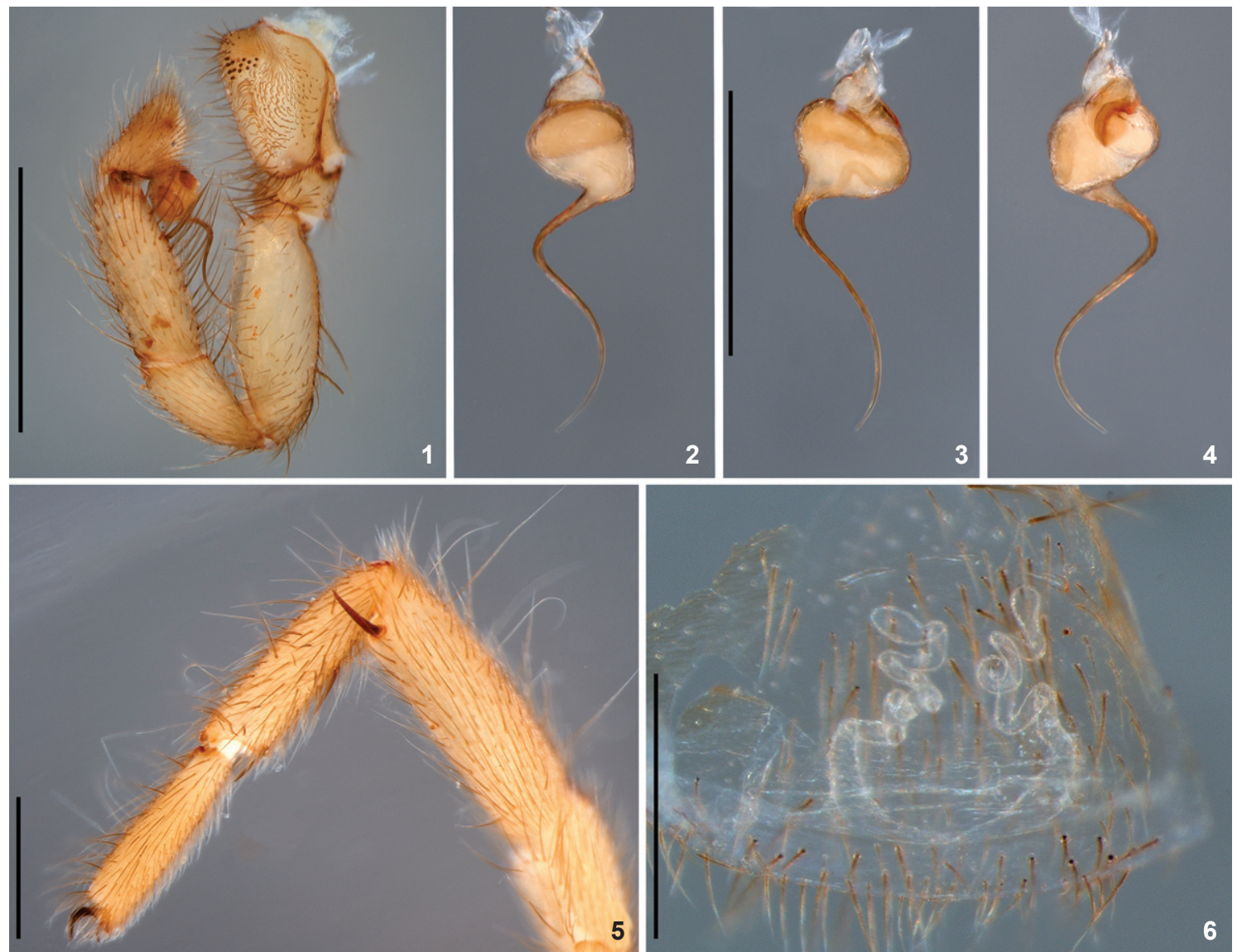

Figures 1-6. Acanthogonatus minimus sp. nov.: (1-5) male: (2-5) holotype: (1-4) left palp: (1) maxilla, tibia and palpal bulb, prolateral view; (2) retrolateral view; (3) ventral view; (4) prolateral view; (5) leg I, retrolateral view; (6) spermathecae paratype (IBSP 112980), dorsal view. Scale bars: Figs. (1): $1 \mathrm{~mm}$; (2-6): 0,5 mm.

Diagnosis. Acanthogonatus minimus sp. nov. differs from remaining species of the genus by the male palpal bulb with very long and twisted embolus ca. of $2 / 3$ length of palpal tibia (Figs. 1-4, 13) and spermatheca with long and twisted duct (Fig. 6), anterior eyes row recurved and T-shaped fovea (Figs. 7, 11).

Description. Male (holotype). Carapace dark yellow, with light pubescence, legs light yellow (Fig. 7). Abdomen dorsally light yellow with two groups of anterior stains, with brown chevron and ventrally light yellow, with light pubescence (Fig. 7). Total length 4.22. Carapace 1.85 long, 1.45 wide, with very short 0.10 and slightly procurved fovea. Abdomen 1.95 long, 1.10 wide. Thoracic region flat. Clypeus absent. Eye tubercle weakly elevated, wider than long (Fig. 7). Eye group trapezoidal. Anterior and posterior eye rows recurved (Fig. 7). Eye sizes: AME 0.10, ALE 0.14, PME 0.09 and PLE 0.12. Chelicerae with seven teeth in prolateral row, with ca. 20 basal smaller teeth (Figs. 9, 16) and rastellum weak, formed by thin setae (Figs. 8, 9, 16). Intercheliceral tumescence very small, pale yellow, covered by ca. nine thin, short, sparse setae (Figs. 9, 16, 17). Labium 0.14 long, 0.30 wide, with no cuspules (Fig. 8). Maxilla subquadrate with 18 blunt cuspules on internal basal angle (Figs. 8, 19, 20). Serrula poorly developed, with ca. 15 teeth (Fig. 18). Sternum slightly circular 1.04 long, 0.85 wide, slightly domed. Six sternal sigilla small, oval, very shallow, inconspicuous (Fig. 8). Palp: measurements: femur 0.85/patella 0.55/tibia 0.70/cymbium 0.40/ total 2.50; spination: femur d0-1-1-1-1-0, tibia r0-0-0-1p-1p. Legs: measurements: I: femur $1.35 /$ patella $0.80 /$ tibia $1.00 /$ metatarsus 0.90/tarsus 0.60/total 4.55; II: 1.05/0.75/0.75/0.70/0.50/3.75; III: 1.05/0.60/1.10/0.95/0.55/4.25; IV: 1.35/0.75/1.05/1.20/0.57/4.92; spination: I: femur d1-1-1-1-0, tibia v1r megaspine (Fig. 5), metatarsus v0-1r-0-2ap; II: femur d1-1-1-1, tibia v0-1-0, metatarsus v0-1r0-2ap; III: femur d1-1-1-2, patella p0-1-1, r0-1-0, tibia d0-01-0, v0-1r-0, p0-1-0, r0-1-0, metatarsus d1r-1p-0-2-0, v2-0-1r-1p0-3ap, p0-1-0-1-0, r0-1-0-0; IV: femur d1-1-1-0, patella r0-1-0, tibia p0-1-0-1-0, rp0-1-0-1-0, metatarsus d2-1p-0-2, v1p-1p-0-3ap, p0-1-0-1-0, r0-1-0-0-0. Slightly thickened femora III (Fig. 7). Metatarsal preening combs: III: 4 VR, 5 VP; IV: 4 VR, 3 VP. Tarsi I-IV not flexible. Scopula on tarsi I-II and $1 / 4$ of metatarsi I-II. 

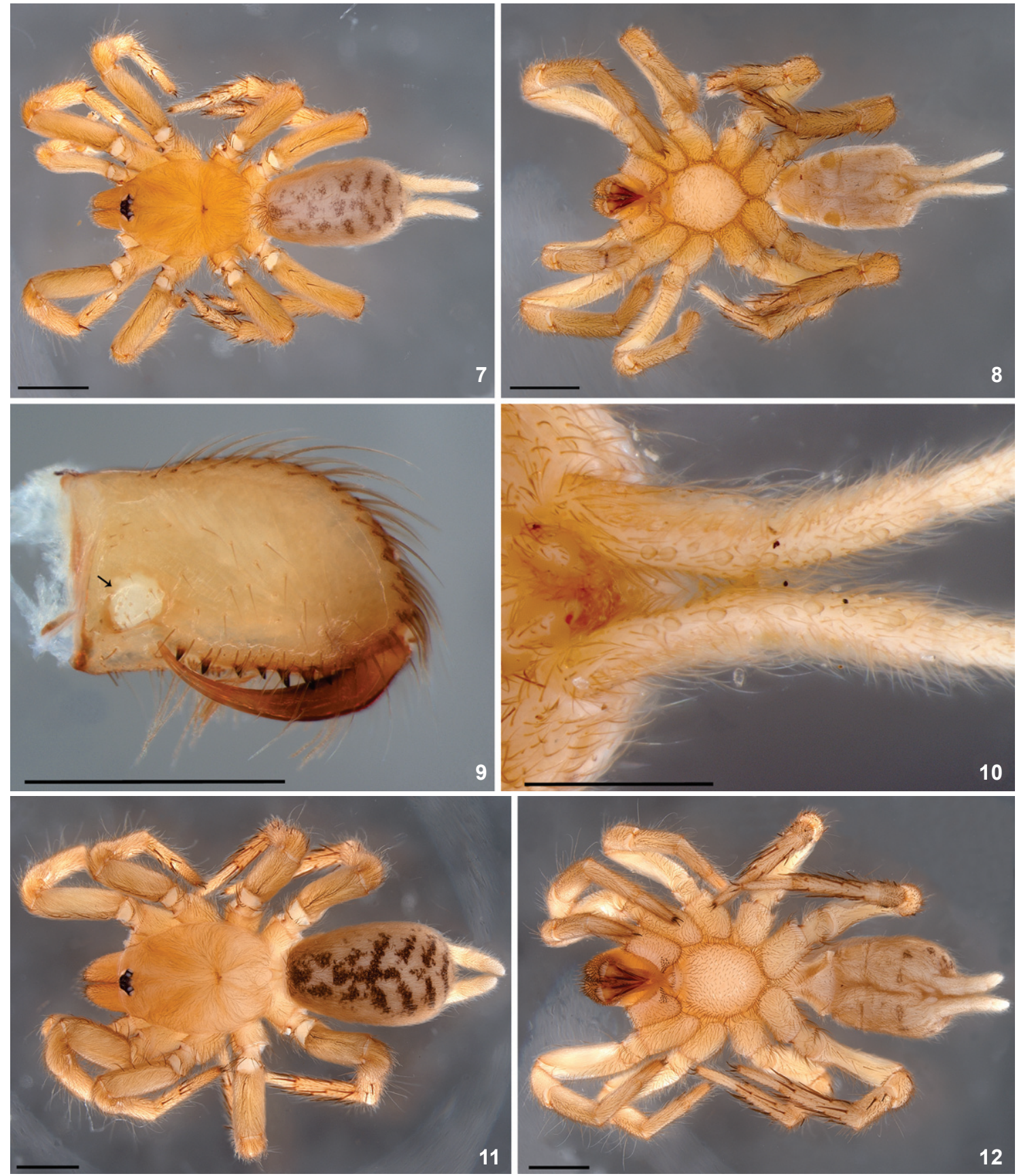

Figures 7-12. Acanthogonatus minimus sp. nov., male: $(7,8,10)$ holotype: (7) body, dorsal view; (8) body, ventral view; (9) left chelicera, arrow shows intercheliceral tumescence, prolateral view; (10) spinnerets, ventral view; (11-12) female paratype (IBSP 112980): (11) body, dorsal view; (12) body, ventral view. Scale bars: 7-8, 11-12 =1.00 mm; $9=0.25 \mathrm{~mm}, 10=0.50 \mathrm{~mm}$.

Scopula on tarsi I-II undivided, very light (Fig. 24) and symmetric. Scopula on tarsi III-IV absent. STC large with double row of teeth. Teeth on tarsal claws: I-II: $13,10,10$, 9; III: 5, 5, 4, 5; IV: 6, $4,5,6$. ITC on all legs (Figs. 5, 24, 25). Trichobothria with rounded, broad, elevated base and 14-16 developed ridges (Figs. $22,23)$. Tarsal organ rounded, slightly elevated with two concentric ridges (Fig. 21). Book-lung openings with long narrow slit. Four spinnerets, PLS: basal segment 0.35 , median segment 
0.45 , apical segment domed 0.50 long (Figs. $7,8,10$ ). PMS with articulate spigots (Figs. 10, 26) and PLS with sparse and enlarged pumpkiniform spigots along inner edge of spinning field and elsewhere covered with articulate spigots (Figs. 10, 27, 28). Cymbium with thin setae, resembling tarsal scopula setae (Figs. 29, 30). Palpal tibia short, narrow (Fig. 1) and with shallow ventral excavation. Palpal bulb almost piriform (Figs. 1-4) with rounded ridges (Figs. 13-15).

Female (paratype IBSP 112980). Coloration as in male (Figs. 11, 12). Total length 5.40. Carapace 2.35 long, 1.75 wide, with very short 0.22 and slightly procurved fovea. Abdomen 2.50 long, 1.55 wide. Thoracic region as in male. Clypeus absent (Fig.
11). Eye tubercle weakly elevated, wider than long. Eye group trapezoidal. Anterior and posterior eye rows recurved (Fig. 11). Eye sizes: AME 0.10, ALE 0.18, PME 0.10 and PLE 0.15. Chelicerae with nine teeth in prolateral row, with ca. 15 basal smaller teeth and rastellum weak, formed by thin setae. Labium 0.24 long, 0.56 wide, without cuspule. Maxilla subquadrate with 30 cuspules on internal basal angle. Serrula as in male. Sternum slightly oval 1.30 long, 1.10 wide. Six sternal sigilla small, oval, very shallow, inconspicuous (Fig. 12). Palp: measurements: femur 1.00/patella 0.60/tibia 0.70/tarsi 0.70/total 3.00; spination: femur d1-1-1-1-1, tibia v3ap. Legs: measurements: I: femur 1.50/ patella 1.10/tibia 1.05/metatarsus 0.85 /tarsus 0.60/total 5.10; II:

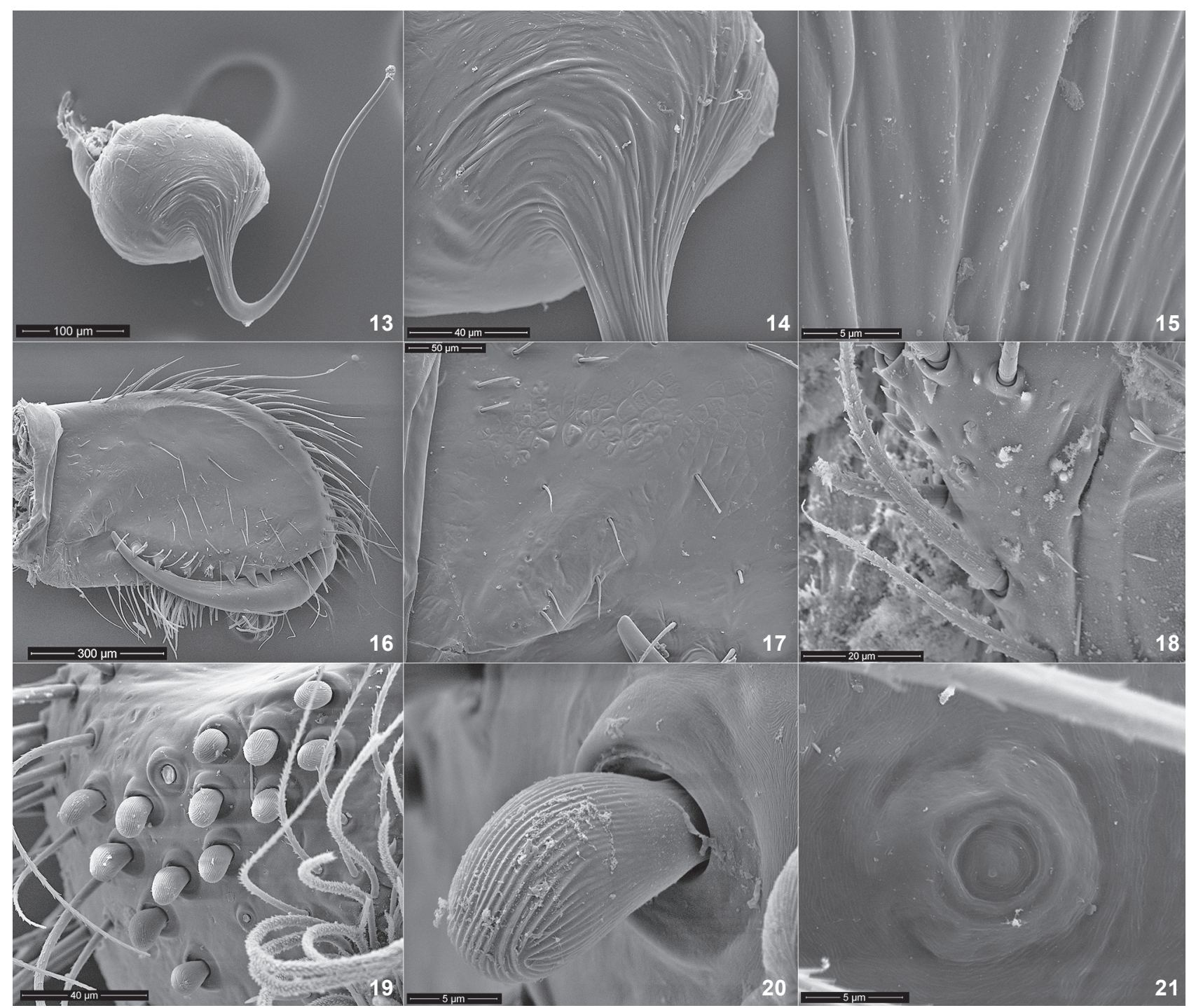

Figures 13-21. Acanthogonatus minimus sp. nov., male: (13-15) left palpal bulb, retro-ventral view: (14-15) detail of rounded ridges; (16-17) left chelicera, prolateral view: (17) detail of intercheliceral tumescence; (18) serrula on maxilla, dorsal view; (19-20) cuspules on maxilla: (20) detail of a cuspule, lateral view; (21) tarsal organ, leg IV, dorsal view. 


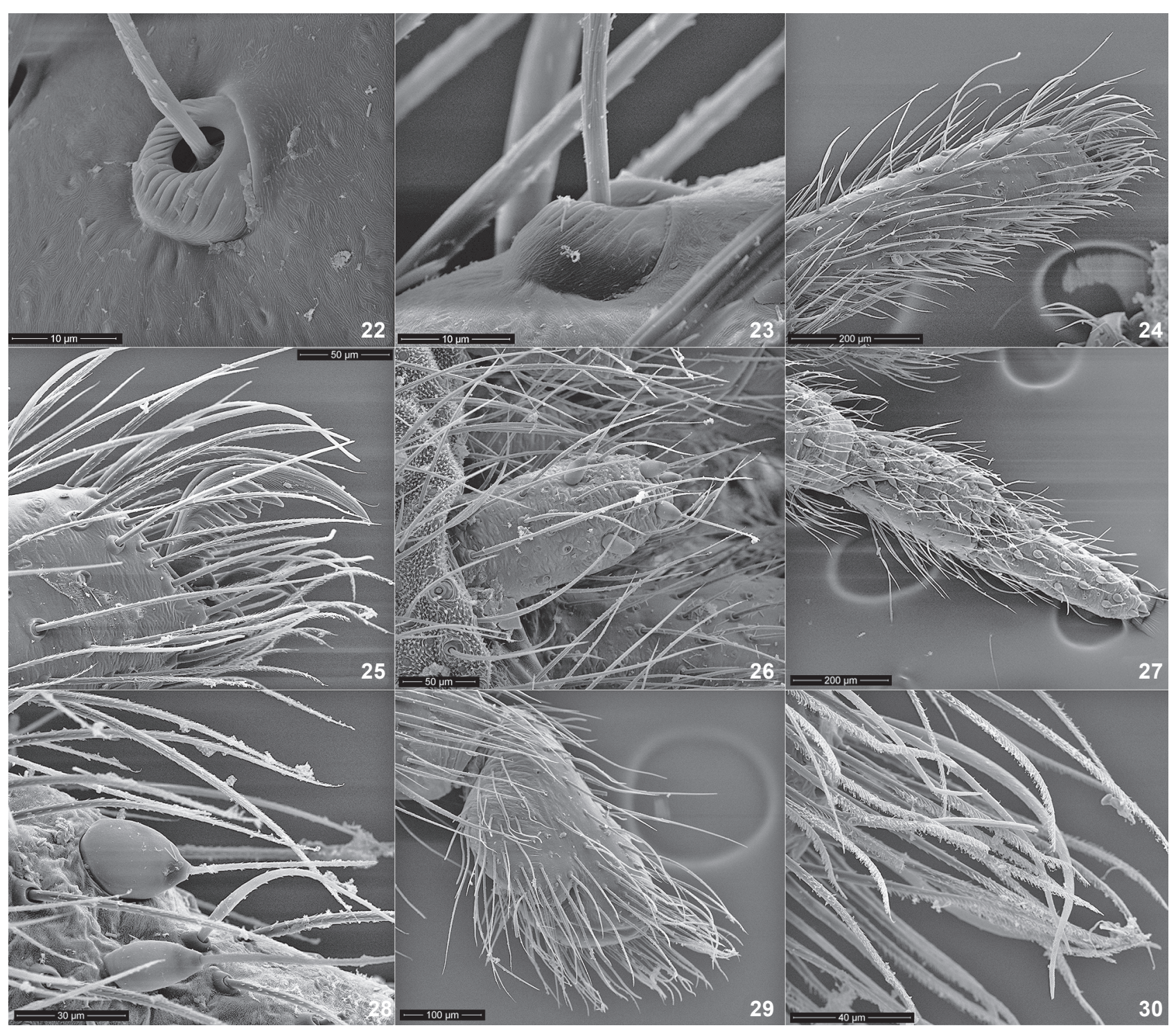

Figures 22-30. Acanthogonatus minimus sp. nov., male: (22-30) left tarsi I: (22, 23) trichobothria; (22) dorso-retrolateral view; (23) retrolateral view; (24-25) tarsi I: (24) retrolateral view; (25) detail of tarsal claws and scopula; (26) spigots in the apex of right posterior median spinneret, ventral view; $(27,28)$ right posterior lateral spinneret, ventral view: $(28)$ pumpkiniform spigot in the inner edge of apical article; $(29,30)$ cymbial left palp, prolateral view; $(30)$ setae on the cymbium, prolateral view.

1.30/0.90/0.80/0.85/0.60/4.45; III: 1.15/0.75/0.70/1.00/0.65/4.25; IV: 1.45/0.90/1.15/1.40/0.70/5.60; spination: I: femur d1-1-1-1, metatarsus v0-1r-0-2ap; II: femur d1-1-1-1-1, metatarsus v0-2-03ap; III: femur d1-1-1-1-1, patella p0-0-1-1, r0-1-0, tibia d0-0-1, v0-1r-0-2ap, p0-1-0-1-0, r0-1-0, metatarsus d1r-2-0-0-2, v1r-0-20-3ap, p0-1-0-1-0; IV: femur d1-1-1-1, tibia v2ap, p0-1-0-1-0, r01-0-1-0 metatarsus d2-0-1r-0-2-0, v20-2-0-3ap, p0-1-0-1-0-0-1, r0-1-0-0. Slightly thickened femora III. Metatarsal preening combs: III: 4 VR, 3 VP; IV: 3 VR, 2 VP. Tarsi I-IV not flexible. Scopula on tarsi I-II undivided, very light and symmetric. Scopula on distal half of metatarsi I. Scopula on tarsi III-IV absent. STC large with double row of teeth. Teeth on tarsal claws: I-II: 10, 10, 10, 10; III: 6, 6, 5, 6; IV: 5, 5, 5, 5. ITC on all legs. Trichobothria and tarsal organ as in male. Book-lung openings as in male. Four spinnerets, PLS: basal segment 0.30 , median segment 0.30 , apical segment domed 0.55 long. Spigots as in male.

Variation. Males $(n=3)$ : total length 4.22-4.40; carapace 1.85-1.90 long; maxillae with 15-18 cuspules. Females $(\mathrm{n}=11)$ : total length 4.00-5.40; carapace 1.80-2.35 long; maxillae with 25-30 cuspules. 
Additional material examined. BraziL, Rio de Janeiro: Nova Iguaçu (Reserva Biológica do Tinguá, 22³4'28.9'S; 43²4'57.6’W, $400 \mathrm{~m}$ a.s.l.), 2002, E. Folly Ramos et al. coll., 1 female, 5 juveniles (IBSP 166093); male (IBSP utilized in SEM); 5 females (IBSP 166094-166098); 2 females (MNRJ 6804).

Distribution. Known only from the type locality.

Etymology. The specific name is a Latin adjective (= the smallest) referring to the small size of this species.

\section{Diplothelopsinae Schiapelli \& Gerschman, 1967 Chaco Tullgren, 1905}

Identification key for Chaco updated from GolobofF (1995) and FerRETTI (2014)

\section{Males}

Male of C. patagonica Goloboff, 1995 and C. tecka Goloboff, 1995 are unknown.

1. ITC IV present; Brazil (Rio de Janeiro) .... C. tingua sp. nov. $1^{\prime}$. ITC IV absent .. 2

2. Tibial apophysis with five or more spines ........................ 3

2 '. Tibial apophysis with less than five spines ...................... 5

3. Embolus extremely long, bulb in lateral view, abruptly constricted to form embolus (Goloboff 1995: fig. 146D); northern Argentina (Salta and Jujuy) C. obscura Tullgren, 1905

3'. Embolus shorter, bulb more gradually tappering .. 4

4. Embolus as about half longer of total bulb length having short keels on base (Golobofr 1995: fig. 148E); northern Argentina (Tucumán and Catamarca) ..... C. tucumana Goloboff, 1995

$4^{\prime}$. Embolus as about one third or less longer of total bulb length having longer keels on base (Montes De OCA \& Pérez-Miles 2013: fig. 4C); Uruguay (atlantic coast)

C. costai Montes de Oca \& Pérez-Miles, 2013

5. Tibial apophysis with four spines

5'. Tibial apophysis with less than four spines .. 8

6. Palpal bulb with a strongly sinuous spermatic duct (MONTES de Oca \& Pérez-Miles 2013: fig. 3A); Uruguay (atlantic coast) .. C. castanea Montes de Oca \& Pérez-Miles, 2013

6'. Palpal bulb with straighter spermatic duct; Chile ........... 7

7. Maxillary cuspules less than 20 (usually 13). Construct beveled doors (Goloboff 1995: figs. 142, 143), grayish coloration; Chile (Region IV) ....... C. socos Goloboff, 1995

7'. Maxillary cuspules more than 20 (usually 33). Construct thin trap-doors (Goloboff 1995: figs. 151, 152), brownish coloration; Chile (Region V) ......... C. tigre Goloboff, 1995

8. Tibial apophysis with three spines; bulb gradually tapering and embolus bent (Goloboff 1995: fig. 154D, E); northwestern Argentina (San Juan) C. sanjuanina Goloboff, 1995
8'. Tibial apophysis with two spines; bulb more abruptly tapering and embolus straight (FERRETTI 2014: fig. 2B); central western Argentina (Mendoza) ...... C. ansilta Ferretti, 2014

\section{Females}

1. ITC IV present; Brazil (Rio de Janeiro) .... C. tingua sp. nov.

1'. ITC IV absent) 2

2. Spermathecae a single undivided tube (sometimes spiraled); northwestern Argentina and Uruguay ............................. 3

2 '. Spermathecae a single tube with a basal protuberance ... 6

3. Spermathecae very long (Goloboff 1995: fig. 147E, F); northern Argentina (Salta and Jujuy) C. obscura

3'. Spermathecae short 4

4. Spermathecae with reniform fundus (Montes de Oca \& PérezMiles 2013: fig. 3E); Uruguay (atlantic coast) .. C. castanea

4'. Spermathecae with subspherical fundus .......................... 5

5. Spermathecae with a sinuous neck (Montes de Oca \& PérezMiles 2013: fig. 4E); Uruguay (atlantic coast) ....... C. costai

5'. Spermathecae with a straight neck (Goloboff 1995: fig. 148A, B); northern Argentina (Tucumán and Catamarca).

C. tucumana

6. Pseudo preening combs present; metatarsus IV with numerous strong spines on superioanterior face; southern Argentina (Chubut) C. tecka

6'. No pseudo preening combs; metatarsus IV with few spines on superoanterior face. 7

7. Sternal sigilla almost inconspicuous; color yellowish light; Argentina .. 8

7'. Sternal sigilla normal, maxilla with medium number of cuspules (15-50), color brownish or ash gray; Chile .... 10

8. Maxillae with few cuspules (6-10), labium with few cuspules irregularly arranged, very light pubsescence .... ... 9

8'. Maxillae with medium number of cuspules (about 12); labium with 8 cuspules in transverse line; northern Argentina (San Juan) C. sanjuanina

9. Femora normal, spermathecae with sinuous neck and subspherical fundus (Goloвоғғ 1995: fig. 156C); southern Argentina (Chubut)

C. patagonica

$9^{\prime}$. Femora incrassate, spermathecae with straight and very long neck and fundus not well differentiate (FerRetTi 2014: fig. 2D); central western Argentina (Mendoza) C. ansilta

10. Spermathecae with very short and thin neck and well developed subspherical fundus (Goloboff 1995: fig. 150B), construct thin trap-doors, brownish coloration; Chile (Region V) C. tigre

10'. Spermathecae with long neck and less developed fundus (GolobofF 1995: fig. 153B), construct beveled doors, grayish coloration; Chile (Region IV) ..... C. socos 

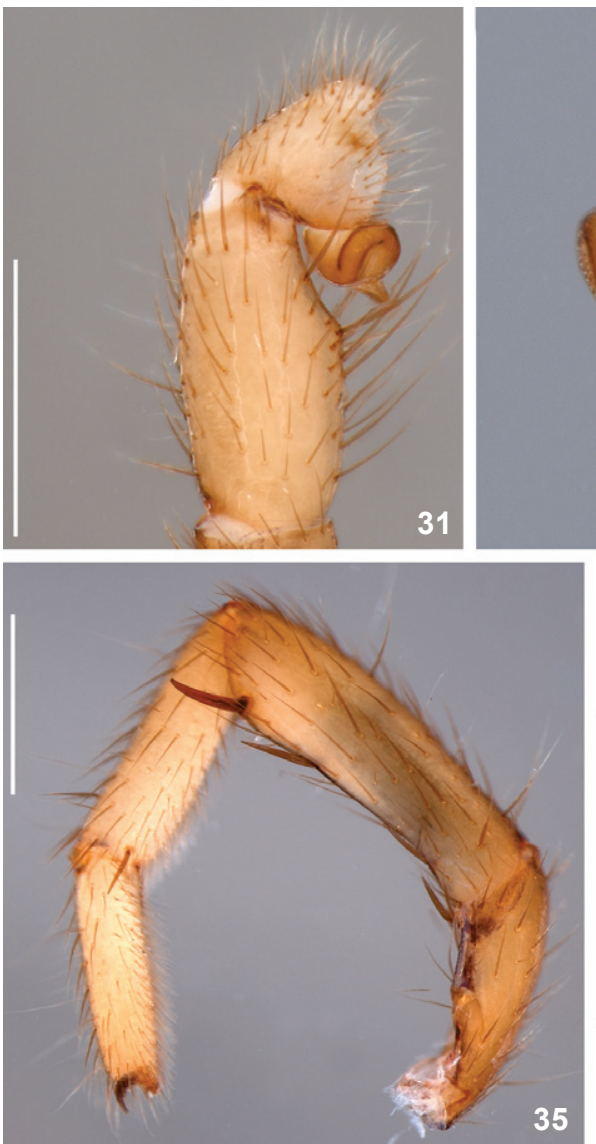

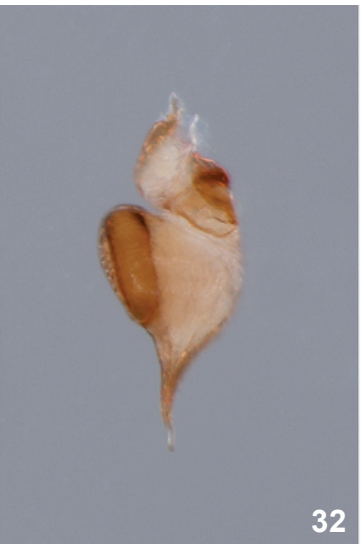

32

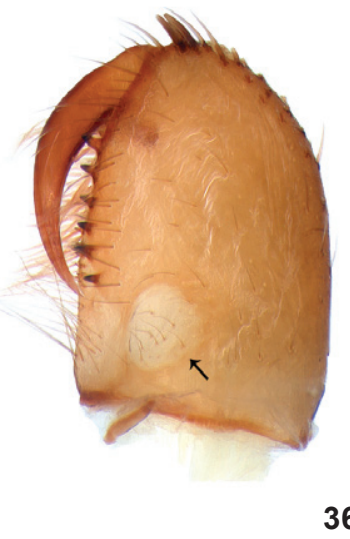

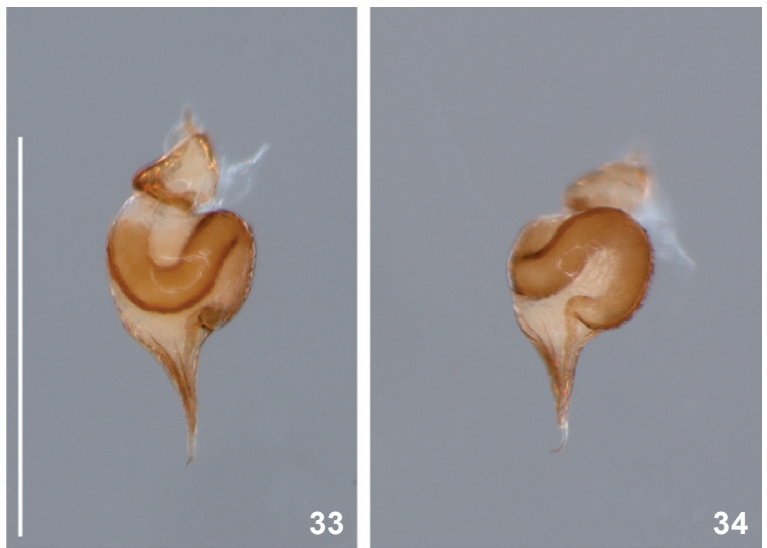

36

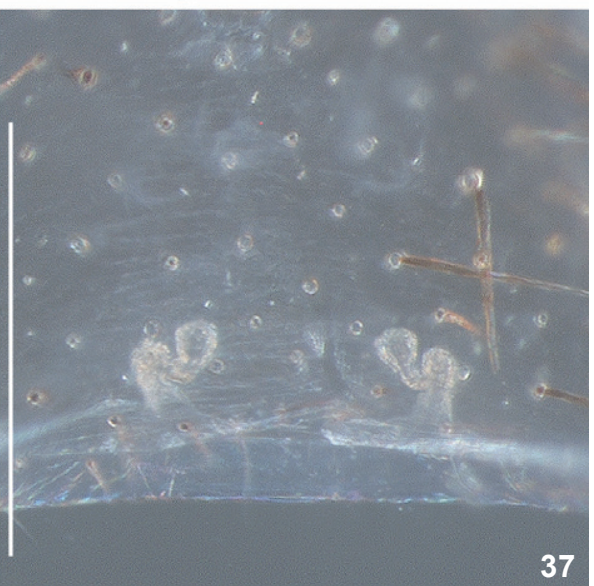

Figures 31-37. Chaco tingua sp. nov.: (31-36) male: (31-35) holotype: (31-34) left palp: (31) palpal tibia and cymbium, prolateral view; (32) prolateral view; (33) retrolateral view; (34) ventral view; (35) leg I, retrolateral view; (36) right chelicera, arrow shows intercheliceral tumescence, prolateral view; (37) spermathecae paratype (IBSP. 03 out 2002), dorsal view. Scale bars: Figs. (31-36): 0,5 mm; (36): 0,25 mm.

\section{Chaco tingua sp. nov.}

Figs. 31-62

Types. Male holotype from BRazIL, Rio de Janeiro: Nova Iguaçu (Reserva Biológica do Tinguá, 22³4'28.9'S; 4324'57.6'W, 400 m a.s.l.), 2002, E. Folly Ramos et al. leg. (IBSP 166099). Paratypes with the same data as holotype, female (IBSP 166100); female (IBSP 166101); male (IBSP 166102); male and female (MNRJ 6805); male and female (MZSP 67297); male and female (MCN 52060).

Diagnosis. Chaco tingua sp. nov. differs from remaining species of the genus by the retrolateral megaspine on tibia I (Figs. 35, 53), palpal embolus tip hook-shaped (Fig. 34), ITC on all legs, absence of pubescence on the carapace and legs (Figs. 38, 40).

Description. Male (holotype). Carapace brown, legs brownish yellow, with no pubescence (Fig. 38). Abdomen dorsally brown with one anterior stain and five median-posterior yellow bands and ventrally yellow, with light pubescence (Fig. 38). Total length 4.20. Carapace 2.00 long, 1.60 wide, with short 0.11 and procurved fovea (Fig. 38). Abdomen 1.90 long, 1.20 wide. Thoracic region slightly raised. Clypeus absent. Eye tubercle weakly elevated, wider than long (Fig. 38). Eye group trapezoidal. Anterior and posterior eye rows recurved (Fig. 38). Eye sizes: AME 0.16, ALE 0.16, PME 0.11 and PLE 0.10. Chelicerae with five teeth in prolateral row (Figs. 36, 49), with ca. 12 basal smaller teeth and strong rastellum, formed by four very thick short setae, without on raised mound (Figs. 36, 39, 49). Intercheliceral tumescence small, pale yellow, covered by ca. 10 thin, long, sparse setae (Figs. 36, 49, 50). Labium 0.08 long, 0.16 wide, with no cuspules (Fig. 39). Maxilla subquadrate with ca. 20 pointed and elongated cuspules on internal basal angle. Serrula very poorly developed, with ca. five teeth (Fig. 48). Sternum circular 0.48 long, 0.42 wide, domed. Six sternal sigilla small, oval, very shallow, inconspicuous (Fig. 39). Palp: measurements: femur 0.85 /patella 0.45 /tibia $0.55 /$ cymbium 0.30/total 2.15; spination: femur d0-0-0-0-1-1, tibia r0-0-0-1p0. Legs: measurements: I: femur $1.55 /$ patella $0.90 /$ tibia $1.15 /$ metatarsus $0.90 /$ tarsus $0.65 /$ total 5.15; II: 1.35/0.85/0.90/1.00/ 0.65/4.75; III: 1.30/0.75/0.90/1.10/0.50/4.55; IV: 1.80/0.75/1.50/ 

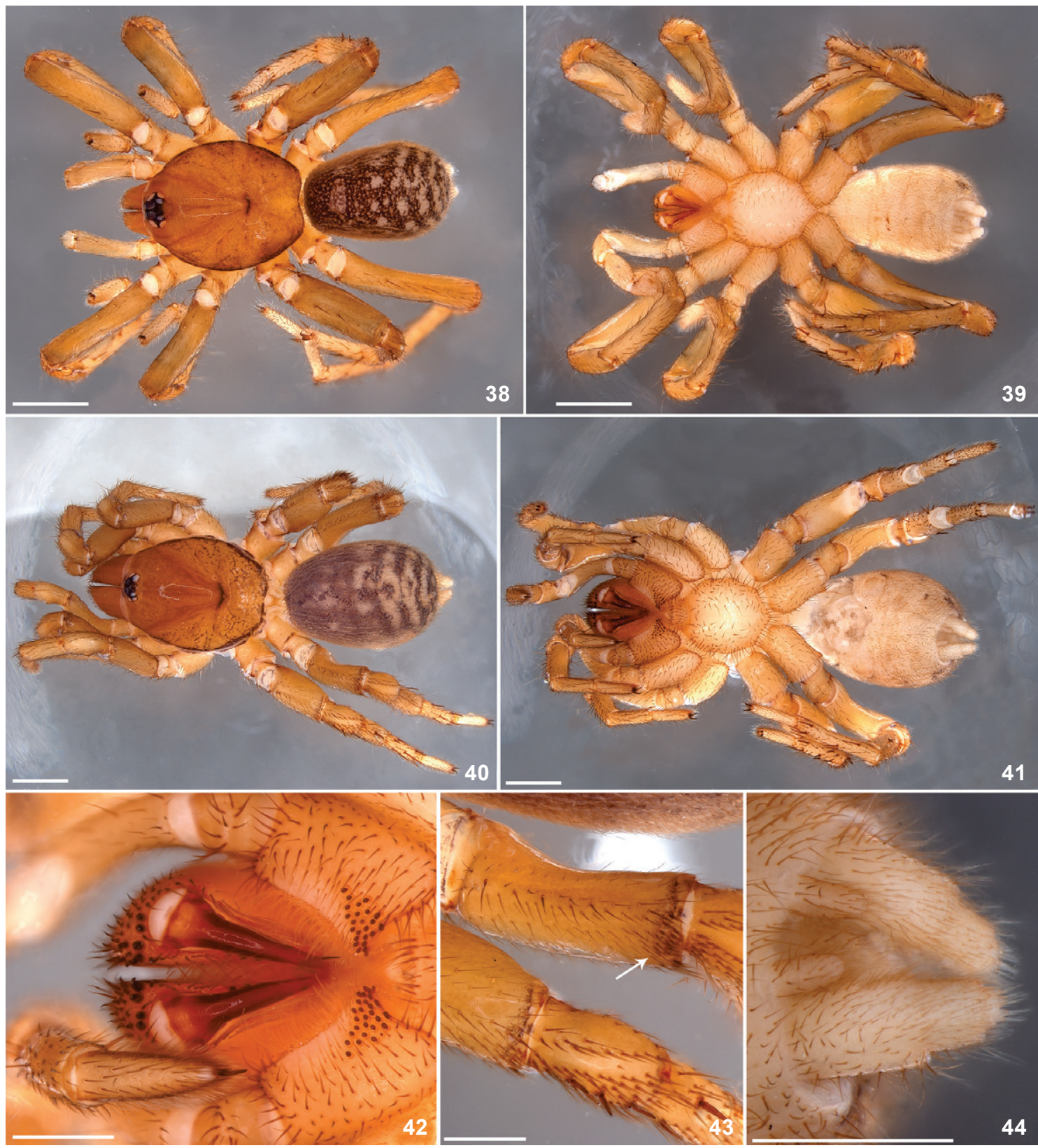

Figures 38-44. Chaco tingua sp. nov.: (38-39) male holotype: (38) body, dorsal view; (39) body, ventral view; (40-44) female paratype (IBSP. 03 out 2002): (40) body, dorsal view; (41) body, ventral view; (42) chelicerae and maxillae, ventral view; (43) legs III-IV, arrow shows the group of thickened setae on femur IV; (44) spinnerets, ventral view. Scale bars: Figs. (40, 41, 43$): 1 \mathrm{~mm}$; $(42,44): 0,5 \mathrm{~mm}$.

1.75/0.90/6.70; spination: I: femur d0-1-1-1, patella v1ap, tibia v1r-0-2-0-2-(1r megaspine, Figs. 35, 53), metatarsus v2ap; II: femur d0-1-1-1, patella 0, tibia v0-1r-0-2ap, p0-1-0-1-0, metatarsus v2ap; III: femur d0-1p-2-1r, patella p0-1-1-1, tibia d0-10, v2ap, p1-1-0, metatarsus d1r-1p-1r-1p-0-2, v3ap, p0-1-0-0; IV: femur d0-1-1-1-0, patella 0, tibia v3ap, p1-0-1-0, r0-1-0-1, metatarsus d1r-1r-0-1p-0-0-2, v0-0-1r-0-3ap, r1-0-1-0-0-0. Thickened femora III (Fig. 38). Group of ca. 10 thickened setae on apical prolatero-dorsal femur IV. Metatarsal preening combs absent. Tarsi I-IV not flexible (Figs. 35, 38-41, 51, 54). Scopula on tarsi I-II and $2 / 3$ of metatarsi I-II (Figs. 35, 51). Scopula on tarsi I-II undivided, very light and symmetric. Scopula on tarsi 


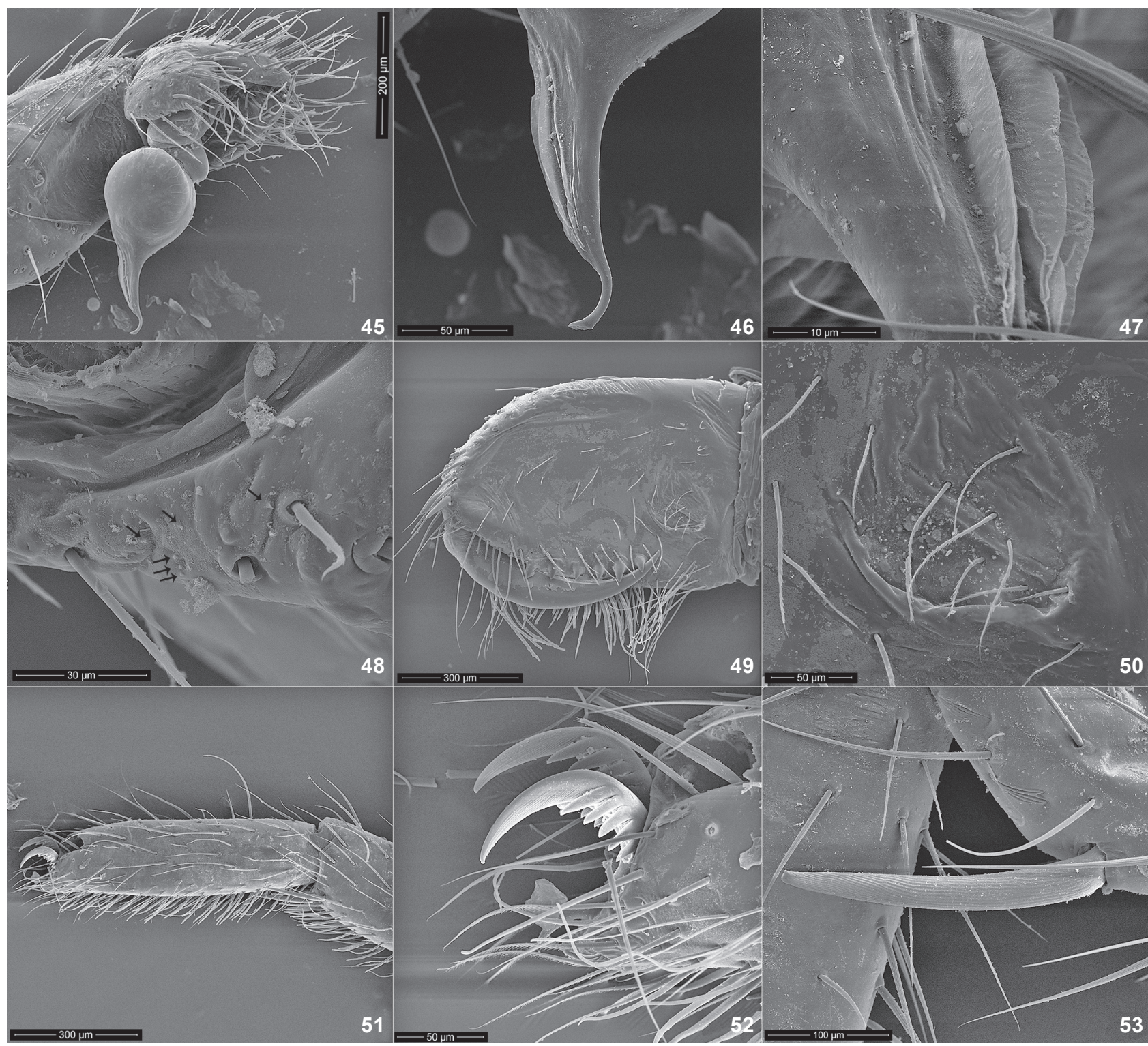

Figures 45-53. Chaco tingua sp. nov., male: (45-47) left palpal bulb, ventral view: (46) keels; (47) keels detail; (48) serrula on maxilla, arrows shows teeth, dorsal view; $(49,50)$ right chelicera, prolateral view: $(50)$ detail of intercheliceral tumescence; $(51-53)$ left leg I, retrolateral view: (51) tarsi I; (52) detail of claws and scopula; (53) detail of megaspine.

III-IV absent (Figs. 38, 54). STC large with double row of teeth (Fig. 52). Teeth on tarsal claws: I and II: 7, 4, 4, 6; III: 6, 4, 4, 6; IV: 6, 4, 5, 6. ITC on all legs (Figs. 52, 55). Trichobothria with oval, broad, elevated base and 10-13 developed ridges (Figs. $51,55,57)$. Tarsal organ oval, slightly elevated with two concentric ridges (Fig. 56). Book-lung openings with long narrow slit. Four spinnerets, PLS: basal segment 0.10 , median segment 0.11 , apical segment domed 0.05 long (Figs. 39, 58). PMS with 3-4 articulate spigots only on apex. PLS covered with sparse articulate spigots, one enlarged on apex of medial article (Fig. 58). Cymbium with thin setae, resembling tarsal scopula setae (Figs. 45, 59). Palpal tibia short, wide (Fig. 31) and with lightly deep ventral excavation (Figs. 31, 45). Palpal tibia excavation and basal region of tegulum with grooves, evidencing a possible stridulatory apparatus (Fig. 45). Palpal bulb piriform with six keels and short and curved embolus (Figs. 32-34, 45-47).

Female (paratype IBSP 166100). Coloration as in male (Figs. 40,41 ). Total length 5.80. Carapace 2.50 long, 2.00 wide, with 


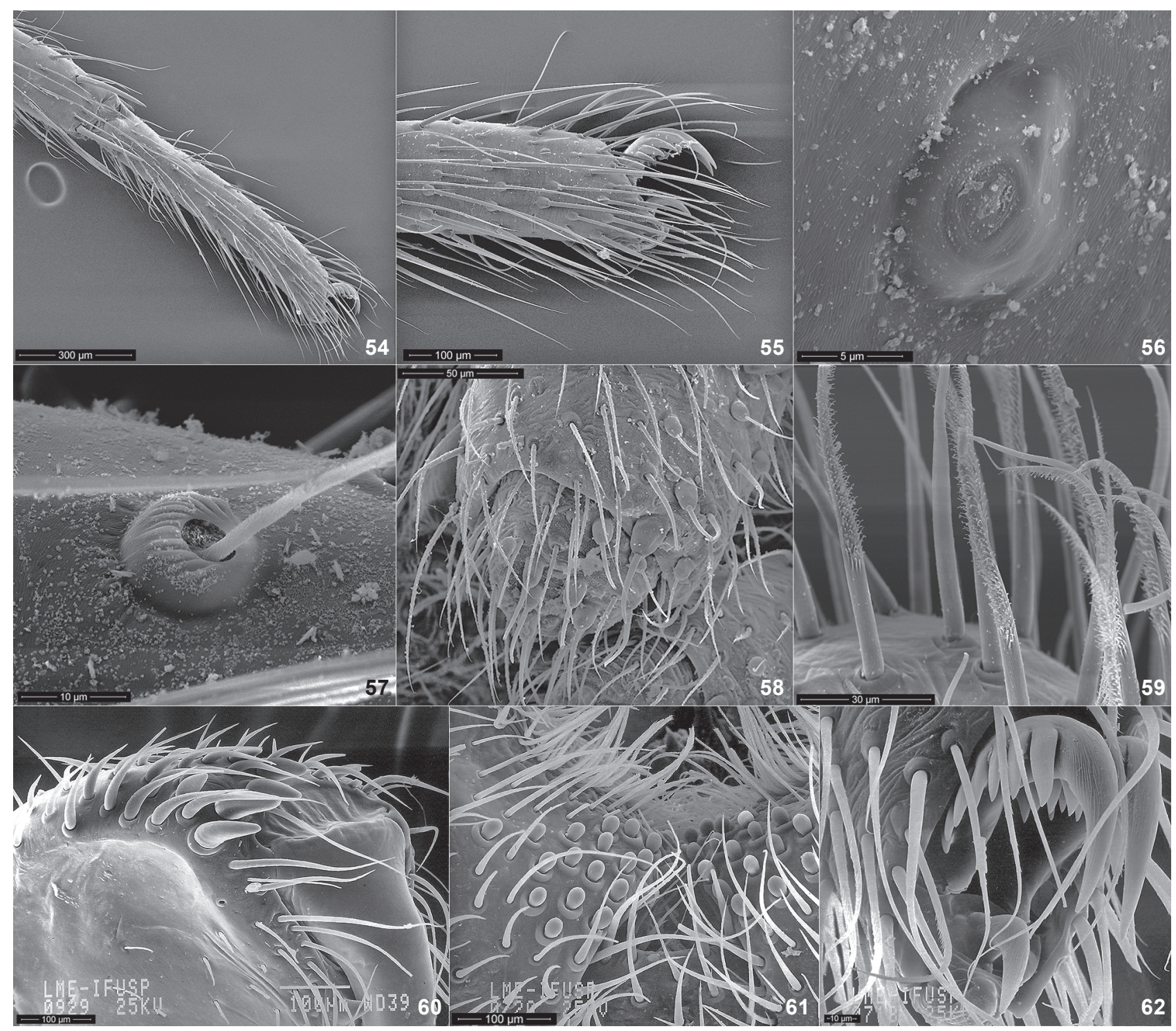

Figures 54-62. Chaco tingua sp. nov.: (54-59) male: (54-57) left tarsi IV: (54-55) prolateral view; (56) tarsal organ, dorsal view; (57) trichobothria, dorso-prolateral view; (58) articulate spigots on apical article of posterior lateral spinnerets; (59) left palp, cymbial setae, prolateral view; (60-62) female: (60) left chelicerae, rastellum, ventral view; (61) cuspules on maxillae, ventral view; (62) claws of tarsi IV, retrolateral view.

short 0.20 and procurved fovea (Fig. 40). Abdomen 2.65 long, 1.90 wide. Thoracic region as in male. Clypeus absent. Eye tubercle weakly elevated, wider than long (Fig. 40). Eye group trapezoidal. Anterior and posterior eye rows recurved (Fig. 40). Eye sizes: AME 0.11, ALE 0.16, PME 0.10 and PLE 0.11. Chelicerae with seven teeth in prolateral row, with ca. 12 basal smaller teeth and strong rastellum formed by five very thick short setae, without on raised mound (Figs. 41, 42, 60). Labium 0.5 long, 0.6 wide, without cuspule. Maxilla with 28 blunt cuspules on internal basal angle (Figs. 41, 42, 61). Serrula absent. Sternum slightly oval 1.5 long, 1.25 wide. Six sternal sigilla small, oval, very shallow, inconspicuous (Fig. 41). Palp: measurements: femur 1.00/ patella $0.62 /$ tibia $0.65 /$ tarsi $0.6 /$ total 2.87 ; spination: tibia v3ap. Legs: measurements: I: femur $1.25 /$ patella $0.85 /$ tibia $0.95 /$ metatarsus 0.70/tarsus 0.55/total 4.3; II: 1.25/0.80/0.75/0.65/0.55/4.00; III: 1.25/0.80/0.80/0.80/0.6/4.25; IV: 1.75/1.00/1.25/1.40/0.80/6.2; spination: I: metatarsus v2ap; II: femur metatarsus v0-1-0-2ap; III: patella p0-1-1-1, tibia d0-1-0, p0-1-0-0, metatarsus d1r-2-1p0-2, v3ap, p0-1-0-0; IV: tibia p0-1-0, metatarsus d1r-1p-0-2, v4ap, p0-1-0-0. Thickened femora III (Fig. 40). Group of ca. 10 thick- 
ened setae on apical prolatero-dorsal femur IV (Figs. 40, 43). Metatarsal preening combs absent. Tarsi I-IV not flexible (Fig. 41). Scopula on tarsi I-II undivided, very light and symmetric. Scopula on distal half of metatarsi I. Scopula on tarsi II-IV absent. STC large with double row of teeth (Fig. 62). Teeth on tarsal claws: I: 9, 5, 5, 9; II: 6, 5, 5, 7; III: 6, 4, 4, 6; IV: 5, 3, 4, 5. ITC on all legs (Fig. 62). Trichobothria and tarsal organ as in male. Book-lung openings as in male. Four spinnerets, PLS: basal segment 0.20 , median segment 0.16 , apical segment domed 0.04 long (Figs. 41, 44). Spigots as in male. Spermatheca with a single receptacula arising from prolateral border of the long basal dome (Fig. 37).

Variation. Males $(n=10)$ : total length 3.85-4.20; carapace 1.60-2.00 long; maxillae with $18-20$ cuspules. Females ( $\mathrm{n}=$ 20): total length 4.50-5.80; carapace 1.00-2.50 long; maxillae with 22-28 cuspules.

Additional material examined. BRAzIL, Rio de Janeiro: Nova Iguaçu (Reserva Biológica do Tinguá, $22^{\circ} 34^{\prime} 28.9^{\prime} \mathrm{S} ; 43^{\circ} 24^{\prime} 57.6^{\prime} \mathrm{W}$, $400 \mathrm{~m}$ ), 2002, E. Folly-Ramos et al. coll., 5 males (IBSP 166103166107); 54 females, 7 juveniles (IBSP 166108); 6 females, pitfall traps (IBSP 166109-166112); 1 male and 4 females (MNRJ 6806); 2 females, pitfall traps (MZSP 67298); 2 females, pitfall traps (MCN 52061).

Distribution. Known only from the type locality.

Natural history. Two females were observed in captivity. They built a horizontal silk-lined burrow on surface of soil with depth of ca. $12 \mathrm{~mm}$ and closed by a thin trap-door.

Etymology. The specific name is a noun in apposition taken from the type locality.

\section{Ecological data}

A total of 1,613 spiders (1,163 Araneomorphae and 450 Mygalomorphae) were collected in leaf litter with Winkler extractor over the course of 12 months, during four field expeditions at the Reserva Biológica do Tinguá. Of these, 436 spiders (15 males, 84 females, 351 juveniles) belong to two new Nemesiidae species, Acanthogonatus minimus sp. nov. and Chaco tingua sp. nov.

Were recorded 22 individuals of $A$. minimus sp. nov., three males (registered in summer), 11 females (seven in the spring collections) and eight juveniles. Since we only collected a few specimens of $A$. minimus sp. nov. it is not possible to draw conclusions on the fenology of the species, but that is sufficient data to compare it with what we know about other species of the genus. We observed that male and female specimens of Acanthogonatus ericae Indicatti et al., 2008 were collected with pitfall traps in the winter and the spring of southern Brazil (InDiCATTI et al. 2008). In another study using pitfall traps (FERRETTI et al. 2012), males of Acanthogonatus centralis Goloboff, 1995 were recorded when temperatures were medium or low (autumn, end of winter and spring), while females and juveniles were collected when temperatures were high or low (summer and winter).

Contrasting with $A$. minimus, C. tingua sp. nov. was abundant in the samples, totaling 12 adult males (2.89\%), 59 females (14.2\%) and 343 juveniles (83.5\%). The phenology of C. tingua sp. nov. is illustrated in Fig. 63, which shows that the main activity period of males was during the winter (seven individuals), decreasing in the spring (three individuals). These values are statistically significant when the seasons are compared $(\mathrm{KW}=9.173$, $\mathrm{GL}=3, \mathrm{p}=0.0270$ ). However, the data obtained on male abundance are are not conclusive since the sample size is small. During the winter the lowest mean temperature and precipitation were recorded during the sampling period (Fig. 64), showing that the winter is probably the mating period of $C$. tingua sp. nov. If the same parameter is observed among females, an increase in the abundance curve is detected, but without clear activity peaks. A slight increase in activity was observed in the spring, when 22 females were collected, and during the summer, when 16 specimens were collected; these differences, however, are not statistically significance. Our dataset does not show clear activity peaks for females of $C$. tingua sp. nov., but there was a small and yet noticeable increase in activity in the spring and summer, when temperatures and precipitation were higher (Fig. 64).

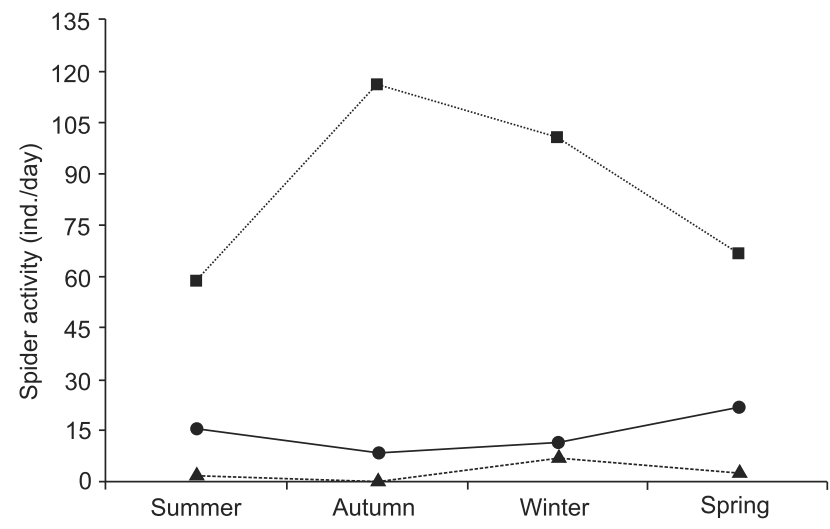

Figure 63. Chaco tingua sp. nov. Phenology based on specimen activity (individuals/day) in Reserva Biológica do Tinguá, Rio de Janeiro using Winkler extractor. ( $(\boldsymbol{\Delta})$ Male, $(\bullet)$ female, ( $(\boldsymbol{\square})$ juvenile.

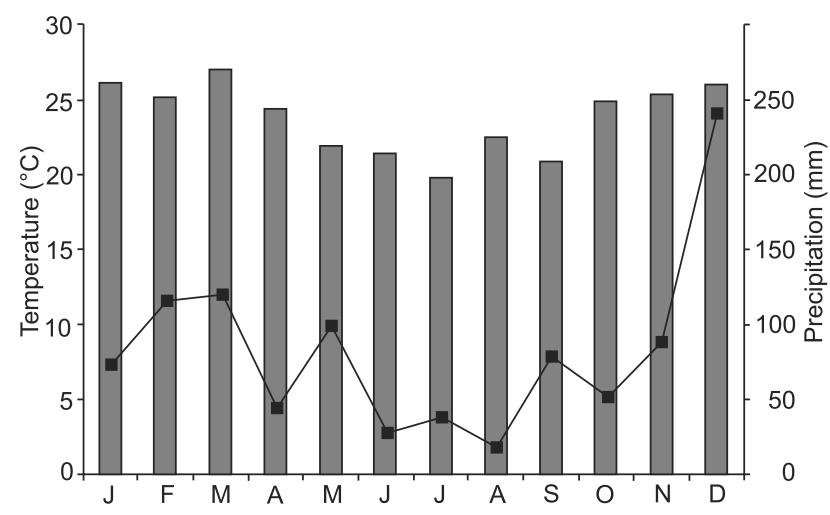

Figure 64. Average temperature and precipitation for the region

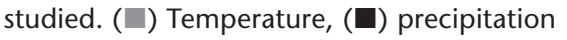


During field captures in the autumn we observed a lack of males and a smaller number of females $(\mathrm{N}=6)$ of C. tingua sp. nov. Juvenile specimens were numerous in samples from the four seasons, with significance values $(\mathrm{KW}=10.902, \mathrm{GL}=$ $3, p=0.0122)$. We suggest that the autumn is the growing period, because newly born Nemesiidae spiders were found forming assemblages in the litter, making their detection easier using the Winkler extractor.

Another observation we have made while looking at the samples obtained with the Winkler extractor is the massive presence of females, five times greater than the number of males. This pattern could be explained by the selectivity of the Winkler trap compared with pitfall traps. The first favors the capture of spiders at an area of a square meter, whereas soil traps such as pitfall traps capture wandering spiders (COSTA \& Pérez-Miles 2002, Candiani et al. 2005, IndicatTi et al. 2005). In addition it is known that female mygalomorphs are more sedentary, whereas males wander a whole lot more (CosTA \& PÉREZMiles 2002, Gonzalez-Filho et al. 2012, Ferretti et al. 2012, Paula et al. 2014).

\section{ACKNOWLEDGMENTS}

We wish to thank Alexander Bernhart for correcting the English in an earlier version of this manuscript; Ronaldo B. Francini Filho for the suggestions on the statistical analyses; three anonymous referees and the editor for their critical comments, which helped us to improve this manuscript; Instituto Chico Mendes de Conservação da Biodiversidade for the permits and the support with field work; Beatriz Maurício for her help with SEM images. This study was supported by Fundação de Amparo à Pesquisa do Estado de São Paulo (grants 2012/ 18287-1 to RPI; 2011/50689-0 to SML and ADB) and Conselho Nacional de Desenvolvimento Científico e Tecnológico (grants 2009/12017-0 to ADB and 479377/2012-0 to RPI).

\section{LITERATURE CITED}

BerTKau P (1880) Verzeichniss der von Prof. Ed. van Beneden auf seiner im Auftrage der Belgischen Regierung unternommen wissenschaftlichen Reise nach Brasilien und La Plata im Jahren 1872-73 gensammelten Arachniden. Mémoires Couronnes de la Academie Royale des Sciences, Lettres et Beaux-Arts de Belgique 43: 1-120.

Candiani DF, Indicatti RP, Brescovit AD (2005) Composição e diversidade da araneofauna (Araneae) de serapilheira em três florestas urbanas na cidade de São Paulo, São Paulo, Brasil. São Paulo, Brasil. Biota Neotropica 5(1a): 1-13. doi: 10.1590/S1676-06032005000200010

Costa FG, Pérez-Miles F (2002) Reproductive biology of Uruguayan theraphosids (Araneae, Theraphosidae). Journal of Arachnology 30: 571-587. doi: 10.1636/0161-8202(2002)030[0571: RBOUTA]2.0.CO;2
Delabie JHC, Agosti D, Nascimejnto IC. (2000) Litter ant communities of the Brazilian Atlantic rain forest region, $\mathrm{p}$. 1-17. In: Agosti D, Majer JD, Alonso LT, Schultz TR (Eds). Sampling ground-dwelling ants: case studies from the world's rain forests. Perth, Curtin University School of Environmental Biology, vol. 18.

Ferretti N (2014) Chaco ansilta new species from Mendoza province, Western Argentina (Araneae: Nemesiidae). Anais da Academia Brasileira de Ciências 2014: 1-12. doi: 10.1095/0001-3765201420140226

Ferretti N, Pompozzi G, Copperi S, Pérez-Miles F, Gonzalez A (2012) Mygalomorph spider community of a natural reserve in a hilly system in central Argentina. Journal of Insect Science 12(31): 1-16. doi: 10.1673/031.012.3101

GoloвоғF PA (1995) A revision of the South American spiders of the family Nemesiidae (Araneae, Mygalomorphae). Part I: species from Peru, Chile, Argentina, and Uruguay. Bulletin of the American Museum of Natural History 224: 1-189.

Gonzalez-Filho HMO, Lucas SM, Paula FS, Indicatti RP, Brescovit AD (2012) On the taxonomy of Acanthoscurria Ausserer from Southeastern Brazil with data on the natural history of $A$. gomesiana Mello-Leitão (Araneae, Mygalomorphae, Theraphosidae). International Journal of Zoology 2012: $1-11$.

IBAMA (2006) Plano de Manejo da Reserva Biologica do Tinguá. Brasília, Ministério do Meio Ambiente, 102p. Available online at: http://www.icmbio.gov.br/portal/ images/stories/imgs-unidades-coservacao/rebio_tingua.pdf. [Accessed: 27/VI/2014].

IndicatTi RP, CANDiani DF, Brescovit AD, Japyassú HF (2005) Diversidade de aranhas (Arachnida, Araneae) de solo na bacia do Reservatório do Guarapiranga, São Paulo, São Paulo, Brasil. Biota Neotropica 5(1a): 1-12. doi: 10.1590/S167606032005000200013

INDICATTI RP, LuCAS SM (2005) Description of a new genus of Nemesiidae (Araneae, Mygalomorphae) from the Brazilian Cerrado. Zootaxa 1088: 11-16.

InDicatTI RP, LuCAS SM, OtT R, BRESCOVIT AD (2008) Litter dwelling mygalomorph spiders (Araneae: Microstigmatidae, Nemesiidae) from Araucaria forests in Southern Brazil, with the description of five new species. Revista Brasileira de Zoologia 25: 529-546. doi: 10.1590/S0101-81752008000300021

Lucas SM, IndicatTi RP (2006) On the genus Psalistopoides MelloLeitão (Araneae, Mygalomorphae, Nemesiidae). Revista Brasileira de Zoologia 23(2): 547-549. doi: 10.1590/S010181752006000200030

LuCAS SM, IndicatTi RP (2010) Description of two new species of Lycinus (Araneae: Nemesiidae). Zoologia 27(3): 425-430. doi: 10.1590/51984-46702010000300015

LuCAS SM, IndicatTi RP, FuKami CY (2005) Redescrição de Prorachias bristowei Mello-Leitão 1924 (Araneae, Mygalomorphae, Nemesiidae). Biota Neotropica 5(1a): 1-6. doi: 10.1590/ S1676-06032005000200019 
Lucas SM, Passanha V, Janini CRV, Indicatti RP (2008) On the genus Neostothis Vellard (Araneae, Nemesiidae). Jounal of Arachnology 36: 472-475. doi: 10.1636/CA07-107.1

Montes de Oca L, Pérez-Miles F (2013) Two new species of Chaco Tullgren from the Atlantic coast of Uruguay (Araneae, Mygalomorphae, Nemesiidae). ZooKeys 337: 7387. doi: 10.3897/zookeys.337.5779

Orsolon-Souza G, Esbérard CEL, Mayhé-Nunes aj, Vargas AB, Veiga-Ferreira S, Folly-Ramos E (2011) Comparison between Winkler's extractor and pitfall traps to estimate leaf litter ants richness (Formicidae) at a rainforest site in southest Brazil. Brazilian Journal of Biology 71(4): 873-880. doi: http://dx.doi.org/10.1590/S1519-69842011000500008

Passanha V, Indicatti RP, Brescovit AD, Lucas SM (2014) Revision of the spider genus Pycnothele Chamberlin, 1917 (Araneae, Nemesiidae). Iheringia 104(2): 228-251. doi: 10.1590/1678476620141042228251

Paula FS, Gabriel R, Indicatti RP, Brescovit AD, Lucas SM (2014) On the Brazilian Amazonian species of Acanthoscurria (Araneae, Theraphosidae). Zoologia 31(1): 63-80. doi: 10.1590/S1984-46702014000100008

Pérez-Miles F, Capocasale RM (1982) Arañas del Uruguay, IV. Hallazgo de una tercera especie del genero Pycnothelopsis: Pycnothelopsis tacuariensis sp. nov. (Araneae, Pycnothelidae).
Comunicaciones Zoologicas del Museo de Historia Natural de Montevideo 147: 1-7.

Pérez-Miles F, Costa FG, Oca LM (2014) Bayana labordai, new genus and species of Nemesiidae (Araneae: Mygalomorphae) from Northern Uruguay and Southern Brazil. Journal of Natural History 48: 1937-1946. doi: 10.1080/00222933.2014.908970

Petrunkevitch A (1925) Arachnida from Panamá. Transactions of the Connecticut Academy of Arts and Sciences 27: 51248.

Platnick NI (2014) The world spider catalog. The American Museum of Natural History, version 15.0, available online at: http://research.amnh.org/iz/spiders/catalog [Accessed: 27/IX/2014] doi: 10.5531/db.iz.0001

RAVEN RJ (1985) The spider infraorder Mygalomorphae (Araneae): Cladistics and systematics. Bulletin of The American Museum of Natural History 182: 1-180.

Raven RJ (1987) A new mygalomorph spider genus from Mexico (Nemesiinae, Nemesiidae, Arachnida). Jounal of Arachnology 14: 357-362.

Tullgren A (1905) Aranedia from the Swedish expedition through the Gran Chaco and the Cordilleras. Arkiv for Zoologi 2(19): 1-81.

ZAR JH (1999) Biostatistical analysis. Upper Saddle River, Prentice-Hall, $4^{\text {th }}, 912 \mathrm{p}$.

Submitted: 10 September 2014

Received in revised form: 31 January 2015

Accepted: 5 February 2015

Editorial responsibility: Ricardo Pinto da Rocha

\section{ERRATA}

Page 127, caption of the Figures 1-6, line 2:

Where read: (2) prolateral view; (3) ventral view; (4) retrolateral view;

Should read: (2) retrolateral view; (3) ventral view; (4) prolateral view;

Page 132, caption of the Figures 31-37, line 2:

Where read: (33) ventral view; (34) retrolateral view;

Should read: (33) retrolateral view; (34) ventral view;

All changes are already incorporated in the online PDF and HTML versions of these articles available at http://www.scielo.br/zool 